\title{
Method of the adaptive decoding of self-orthogonal codes in telecommunication
}

\author{
Juliy Boiko ${ }^{1}$, Ilya Pyatin ${ }^{2}$, Oleksander Eromenko ${ }^{3}$, Mykhailo Stepanov ${ }^{4}$ \\ ${ }^{1,2}$ Department of Telecommunications and Radio Engineering, Khmelnytsky National University, Ukraine \\ ${ }^{3}$ Department of Physics and Electrical Engineering, Khmelnytsky National University, Ukraine \\ ${ }^{4}$ Department of Information Systems and Technologies, Taras Shevchenko National University, Ukraine
}

\begin{tabular}{l} 
Article Info \\
\hline Article history: \\
Received Dec 23, 2019 \\
Revised Mar 7, 2020 \\
Accepted Mar 24, 2020 \\
\hline Keywords: \\
Adaptive algorithms \\
Coder \\
Modulation \\
Noise immunity \\
Telecommunication
\end{tabular}

Article Info

history:

Revised Mar 7, 2020

Keywords:

Adaptive algorithms

Coder

Noise immunity

\begin{abstract}
The methodology description of the adaptive multi-threshold decoding of self-orthogonal codes in the telecommunication channels of information transfer is shown in this paper. The method of multi-threshold decoder modification is described on the basis of adaptive filtration algorithms. Principles of adaptive algorithms application provide for necessary data transmission validity in the case of the multi-threshold decoding are explored. The graphic charts of multi-threshold decoders noise immunity of self-orthogonal block and convolutional codes are presented. It is determined the coding gain (CG) for multi-threshold decoding schemes. The result of research conducted in the course of the paper is to develop a set of scientifically grounded theoretical positions and practical recommendations and proposals for the development of mechanisms of formalization of description of method of increasing of noise immunity of telecommunication systems transmitting information to the synthesis and improving receiver circuit modulated signals on the theory and practice the use of signal-code constructions (SCC) when deciding maximize system capacity information transmission in the presence of noise.
\end{abstract}

Copyright @ 2020 Institute of Advanced Engineering and Science. All rights reserved.

\section{Corresponding Author:}

Juliy Boiko,

Department of Telecommunications and Radio Engineering,

Khmelnytsky National University,

11, Instytuts'ka str., Khmelnitsky, 29016, Ukraine.

Email: boiko_julius@ukr.net

\section{INTRODUCTION}

Noise immunity coding is inseparable part of the modern digital telecommunication systems of information transfer. Necessary quality of discrete information which is passed to telecommunication channels is provided for due to error correction that are arised up in transmission channels [1-4]. A code theory is the applied science. It is integrated in the tasks, which decide in particular the facilities of telecommunications, radiolocation, measuring, computing and managing engineering. In this case, the fact of application possibility of the received results has an important value, their competitiveness in comparing to the uncoded methods of error protection. Practical achievements of code theory are well-known nowadays [5-11].

At the present stage of telecommunication technologies development, such methods of coding have got the widest application: Low-density parity-check code (LDPC), Hamming code, Syndrome vector, convolutional code, Turbo codes and others. Development of telecommunication standards and communication technologies, such as 4G (LTE), 5G, IEEE 802.3an (10G Ethernet), IEEE 802.15.3c, IEEE $802.11 \mathrm{n}$ (WiFi), IEEE 802.16e (WiMAX), satellite communication and data communication systems, telemetry, digital television systems of DVB-S, DVB-S2X, ISDB-Tmm, DVB-T2 formats and others determines researchers to the necessity of search of coding/decoding methods, able to function for rates which arrive tens of $\mathrm{GHz}$ at the simultaneous providing of necessary level of noise immunity [12-14]. 
The review of literary sources allows to establish, that multi-threshold decoders of self-orthogonal codes is the effective enough method of decision of increase quality decoding and its rate tasks [1, 15-17]. The papers of the authors $[1,2,7]$, represent detailed description of methods for multi-threshold decoding. However, the main issues considered in these publications mainly concern to decoding features of structured LDPC-codes, turbo-codes, BCH-codes and others [9]. In papers [10, 14, 15] authors provide recommendations on the features of iteration process in decoding, and consider efficient multi-threshold decoding algorithms for wireless channels [16]. Detailed research of advantages of multi-threshold decoding in a binary erasure channel is provided in [15]. The represented paper complements the research of authors covered in [18-21].

The problem solved in the paper is related to modification of the scheme for self-orthogonal code decoder in order to provide the necessary accuracy in data transmission with minimal redundancy of antijamming code in conditions of hindrance and noise. The main research novelty is related to the idea of building adaptive multi-threshold decoding schemes. The paper deals with the schemes of adaptive multi-threshold decoding convolutional and block codes in telecommunication channels with phase shift keying [11]. The feature of proposed idea is to adaptively change parameters of the encoder and decoder to the state of telecommunication channel, taking into account the interference intensity. This approach will adaptively change the structure of decoder and adjust it to decode a certain set of code, which would have the maximum code speed and provide a given reliability in conditions of strong interference in telecommunication channel.

To solve the problem the synthesis of adaptive decoder of self-orthogonal codes was performed. The mathematical description is given and the recommendations on the choice of adaptive algorithm are developed. The paper presents research results on the proposed multi-threshold decoder in the telecommunication channel with phase shift keying. The paper compares efficiency of the proposed scheme with existing SCC. The energy gain in telecommunication channels with high interference level is determined.

\section{RESEARCH METHOD}

Necessity of solution the immediate theory and practice tasks of noise immunity codes application in telecommunications facilities forms an important scientific and technical task which involves the search of minimization of propagation of errors methods in the case of the multi-threshold decoding. It is necessary to carry out burst errors correction and conduct the synthesis of codes with the effect of minimum propagation of errors which provide high quality of information transfer in the telemetric and telecommunication channels of information transfer. The described tasks are united in the article. It should be noted that major part of task providing of necessary data transmission validity depends exactly upon the algorithm of noiseless coding and more precisely on a decoder. In relation to necessary configuration of SCC for the different standards of information transfer will erect the systematized requirements in a Table 1.

Table 1. Parameters SCC of some telecommunication systems

\begin{tabular}{ccr}
\hline $\begin{array}{c}\text { Telecommunication } \\
\text { frame }\end{array}$ & Mode of code & $\begin{array}{c}\text { Code rate; } \\
\text { (Length of code) }\end{array}$ \\
\hline Satellite transmission & CC+RS & $1 / 2,1 / 3,3 / 4 ;(255,223)$ \\
UMTS/LTE & TC & $1 / 2 ;(640-65536)$ \\
DVB-S2X & LDPC+BCH & $1 / 5,11 / 45,1 / 3,14 / 45,7 / 15,8 / 15 ;(3070-16200)$ \\
DVB-S2 & LDPC+ BCH & $1 / 4,1 / 3,2 / 5,1 / 2,3 / 5,2 / 3,3 / 4,4 / 5,5 / 6,8 / 9,9 / 10 ;(64800)$ \\
DVB-S & CC+RS & $1 / 4,1 / 3,2 / 5,1 / 2,3 / 5,2 / 3,8 / 9,9 / 10 ;(1600)$ \\
DVB-RCS & TC & $1 / 2,1 / 4,1 / 3,2 / 5,3 / 5,2 / 3,3 / 4,4 / 5,5 / 6,8 / 9,9 / 10 ;(1600)$ \\
ISDN-Tmm & CC+RS & $1 / 2,2 / 3,3 / 4,5 / 6,7 / 8 ;(1504,1632)$ \\
\hline
\end{tabular}

In a Table 1 the followings denotations are used: SCC - signal code construction, CC - convolutional code, RS - Reed-Solomon codes, LDPC - low-density parity-check code, BCH - Bose Chaudhuri Hocquenghem codes, TC - turbo codes [2, 6, 9, 10, 16, 17]. Application of algorithms error correction can appear problematic through high complication of decoder which will not have time to produce processing of high-speed information stream. Precisely these circumstances induce to the search of error correction new methods which will have low complication of realization at high efficiency of decoding.

Among codes which admit the threshold decoding, the simplest are the self-orthogonal codes. All self-orthogonal codes assume complete orthogonality. All errors can be corrected with the help of the majority-logic decoding, and their correction is guaranteed by minimum code distance. The method of 
modification of multi-threshold decoder on the basis of adaptive algorithms of filtration is described in the article. Method of application of adaptive algorithms for providing of necessary authenticity of information transfer for multi-threshold decoding is explored.

Serve convolved self-orthogonal code which is used in a telecommunication channel for transmission $k$ connections from binary characters. After a coding the general amount of characters will be evened $n(n>k)$. Let assume that as a result of transferrable of this information in a channel with phase shift keying (binary symmetric channel), decoder in the consequence of presence of noises and hindrances will get

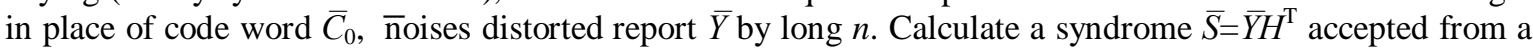
report and for every information character $i_{j}, 1 \leq j \leq k$ will select the plural $\left\{S_{j k}\right\}$ of elements of syndrome with numbers $\left\{j_{k}\right\}$ which will be verifications in relation to character of $u_{j}$. Let this plural contains in quality an element an error $e_{j}$ in every character. The chart of decoder will present in Figure 1.

Additionally enter a binary vector $\bar{D}$ in length $\mathrm{k}$ which will be a differentiating one. Its value will be even a zero in the initial state. In this register mark the changes of information characters with the purpose of memorizing by a decoder accepted report and calculation from a channel in a necessary moment of difference between the accepted report and codeword which is located in information register. Conduct a decoding algorithm by a calculation for $u_{j}$ character taken in an arbitrary way of likelihood function $L_{j}$ which must be dictated by checking of $S_{j k}$ character and $j$ vector component $\bar{D}$ :

$$
L_{j}=\sum_{\{j\}} S_{j k}+d_{j},
$$

where: $L_{j}=$ Likelihood function

$S_{j k}=$ Constituent of character checking

$d_{j}=$ Constituent of additional differentiating vector

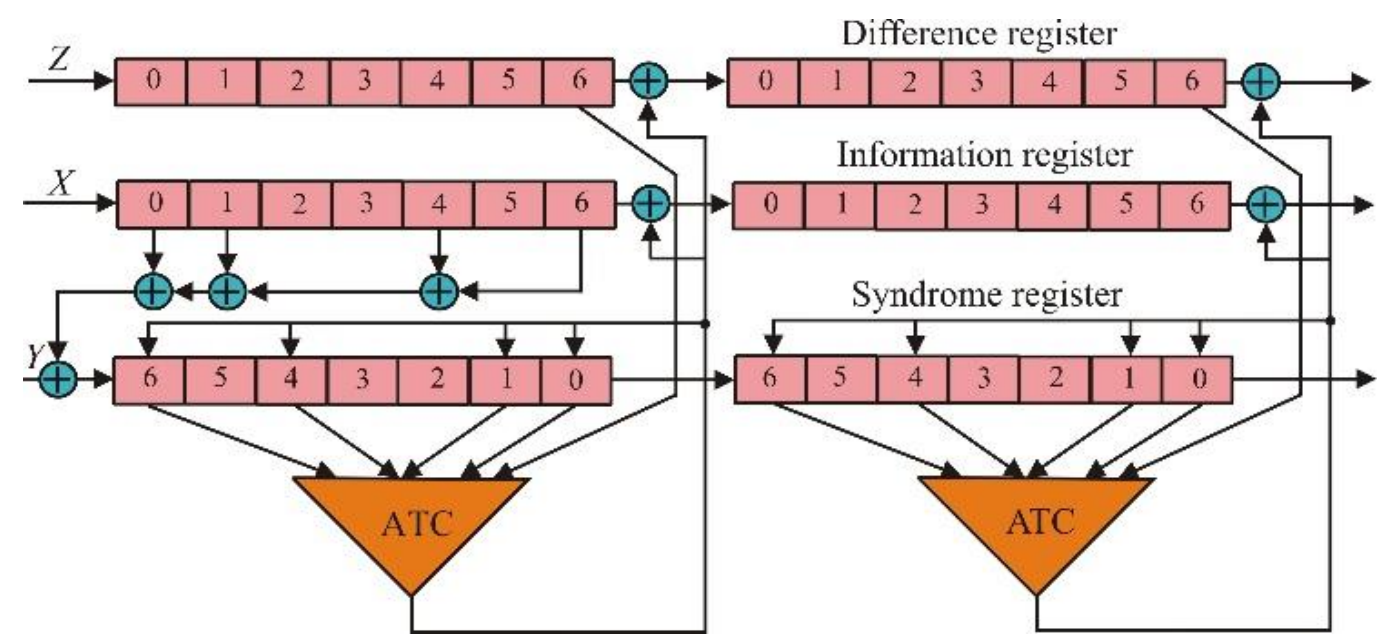

Figure 1. Scheme of multi-threshold decoder of self-orthogonal convolutional codes: $d=5, R=1 / 2, n_{A}=13$, two iterations; ATC - adaptive threshold component

A general amount of terms in a formula (1) must be even $d$ code distance. When $L_{j}>T(T=(d-1) / 2$ is a threshold value), character $u_{j}$, checking $\left\{S_{j k}\right\}$ and character $d_{j}$ will be inverted and other character um $(m \neq j)$ will be garbled. For this character the sum $L_{m}$ etc is calculated in like manner. If $L_{j} \leq T$ then usage passing to recurrent character um at once. Such a condition is being set: implementation of basic step of decoding all $k$ of information report characters can be accompanied by frequent surplus them in a random order.

It is suggested to conduct the value of threshold adaptive, namely, on the first iterations of decoding to increase the size of threshold. In this case a threshold component on the first iterations will bring in less of its own errors in a report which is passed to the next iterations. So, the presented below researches showed that such an approach allows to increase essentially the general decoder scheme efficiency (Figure 1).

Codes with code minimum distance $d$, from 3 to 17 are used for research. Codes will be formed with rates $1 / 2,2 / 3,1 / 3,4 / 6,4 / 7,4 / 8,4 / 9,4 / 10,4 / 11,4 / 12$ in length to 100000 bits. For achievement of optimum decision (Figure 2, curve 5: $R=1 / 2, d=9 ; q=256$ ), a multi-threshold decoder carried out 5-15 decoding iterations of the accepted report. The noise immunity curves are used in Figure 2 and Figure 3 for RS codes with analogical parameters, which are widespread enough in the telecommunication channels [18, 19]. 


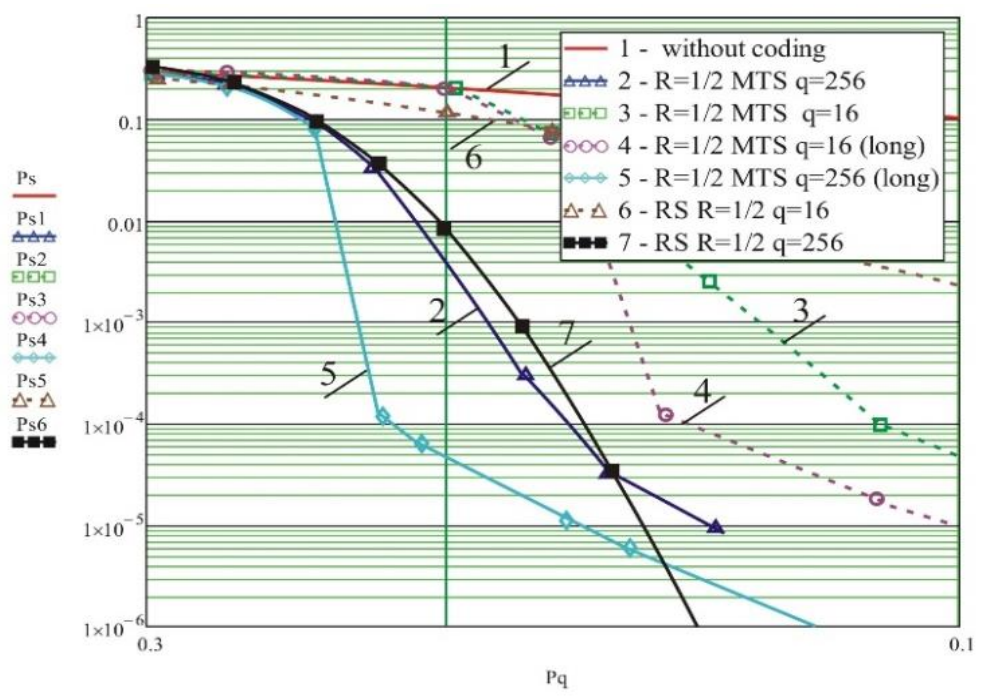

Figure 2. Noise immunity curves of multi-threshold decoder for short and long block self-orthogonal codes: $q$ - is an amount of quantizing levels of demodulator output; $R$ - is code rate (1/2); MTS - multi-threshold scheme; $\mathrm{RS}$ - is a reed solomon code

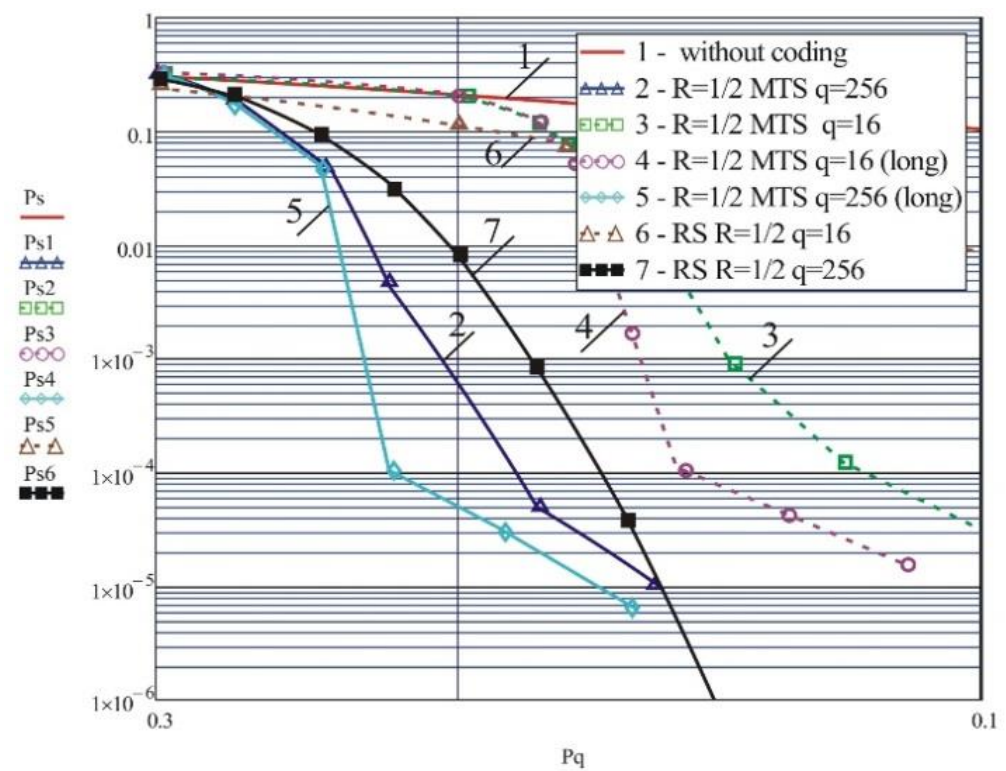

Figure 3. Noise immunity curves of multi-threshold decoder for short and long convolutional self-orthogonal codes: $q$ - is an amount of quantizing levels of demodulator output; $R$ - code rate (1/2);

MTS - multi threshold scheme; RS - is a code of reed solomon

The results of design of multi-threshold decoders in the telecommunication channels with multiposition phase shift keying in the case of exportation of hard decision by a demodulator are presented in Figure 4 and Figure 5. In researching the block and convolved self-orthogonal codes with rates 4/5, 7/8 $(d=9)$ are used in the case of signals employment of type 16QAM and 256QAM (hard decision of demodulator) $[5,20,21]$. Codes in minimum probability of propagation of errors and $n=500$ were used as long codes but comparison was with RS codes [6].

Design results specify that a multi-threshold decoder provides the best descriptions comparing to RS-codes. It is obvious at the small q values due to greater length $\mathrm{n}$ of the used codes and satisfactory enough convergence of decisions of multi-threshold decoder to the optimum decoder decisions (in Figure 2 and Figure 3 the curve of optimum decision isn't shown for clarity). 


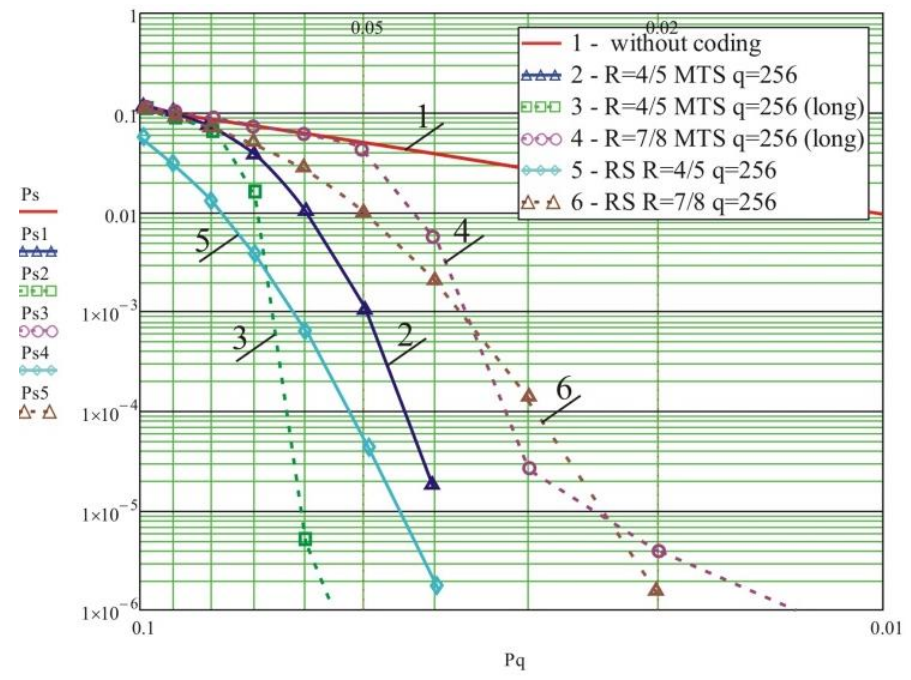

Figure 4. Noise immunity curves of multi-threshold decoder for short and long convolutional self-orthogonal codes: $q$-is an amount of quantizing levels of demodulator output; $R$ - code rate $(4 / 5 ; 7 / 8)$;

MTS - multi threshold scheme; RS - is a code of reed solomon

Schemes are built in coordinates as probability dependence of character $P_{q}$ distortion on middle probability of error on $P_{s}$ character after decoding.

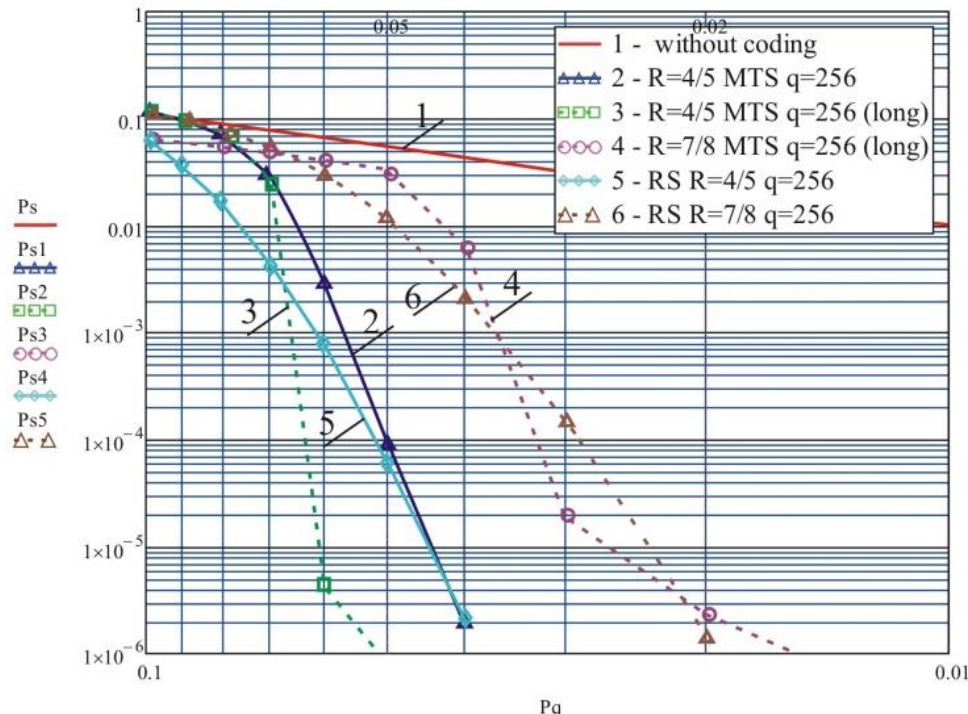

Figure 5. Noise immunity curves of multi-threshold decoder for short and long convolutional self-orthogonal codes: $q$ - is an amount of quantizing levels of demodulator output; $R$ - is code rate $(4 / 5 ; 7 / 8)$;

MTS - multi-threshold scheme; RS - is a code of reed solomon

\section{RESULTS AND ANALYSIS}

The adaptive decoding mechanism is based on the thing that a multi-threshold decoder contains the collection of informative and checking spurs, so it allows to manage surplus by connecting or shutting down of checking spurs.

\subsection{Investigation of adaptive algorithms}

The process of adaptation was corrected to the evaluation by receiver SNR (Signal-to-Noise Ratio) in a telecommunication channel and adaptive choice, based on such an estimation from the certain code 
totality that can have maximum code rate and can provide the given validity. The high noise level in the channel will draw the necessity of powerful code application with the greater number of decoding iterations. Opposite, low level of noises will draw the necessity of less powerful code usage and less number of decoding iterations [6, 15].

The offered algorithm gives the possibility to change adaptive coder and decoder parameters for the state of telecommunication channel, relying on intensity of noises. Algorithm of RLS adaptation (Recursive Least Square) is used for the lead through of adaptation with the purpose of SNR determination in a telecommunication channel [20-24]. Let the entrance discrete random signal $x(t)$ be processed with the nonrecursive discrete filter of $N$ order. The coefficients of this filter can be presented with the vector-column $\mathbf{w}=\left[\mathrm{w}_{0}, \mathrm{w}_{1}, \ldots, \mathrm{w}_{N}\right]^{\mathrm{T}}$. An initial signal of filter will be even:

$$
y(k)=\mathbf{u}^{\mathrm{T}}(k) \mathbf{w},
$$

where: $\mathbf{u}(k)=[x(k), x(k-1), \ldots, x(k-N)]^{\mathrm{T}}=$ Vector-column of content line of filter delay on $k$ step. In addition, a signal $\mathrm{d}(\mathrm{k})$ is exemplary (also random). The error of exemplary signal recreation is evened:

$$
e(k)=d(k)-y(k)=d(k)-\mathbf{u}^{\mathrm{T}}(k) \mathbf{w},
$$

It is necessary to find $w$ filter coefficients which provide the maximal closeness of filter initial signal to exemplary one and minimize the error $e(k)$. As $e(k)$ is also a random process, then accept a mean square as the measure its size. Thus, an optimizing functional is: $J(\mathrm{w})=\overline{e^{2}(k)} \rightarrow \mathrm{min}$. Approach to form of adaptive processing algorithm is used in paper, when during the process of signal acceptance on every duty step the filter coefficients are transferred directly for formula (2). It is connected with unjustified large calculable charges.

$$
\mathbf{w}=\left(\mathbf{U U}^{\mathrm{T}}\right)^{-\mathbf{1}} \mathbf{U d}
$$

Indeed, the size of $\mathbf{U}$ matrix is being constantly increased and every time it is necessary to calculate anew an inverse matrix $\left(\mathbf{U} \mathbf{U}^{\mathrm{T})-1}\right.$. It is possible to shorten the calculable charges if is noticed that one new column entirely is added at every step to $\mathbf{U}$ matrix, and to vector $\mathbf{d}$ is added one new unit. It enables to organize a recursive computation. Recursive estimation update of inverse correlation matrix $\left(\mathbf{U U}^{\mathrm{T})-1}\right.$ is conducted at the use of RLS algorithm and the leading out of formulas is based on the following matrix identity [18]:

$$
(\mathbf{A}+\mathbf{B C D})^{-1}=\mathbf{A}^{-1}-\mathbf{A}^{-1} \mathbf{B}\left(\mathbf{C}^{-1}+\mathbf{D A}^{-1} \mathbf{B}\right) \mathbf{D A}^{-1}
$$

where: $\mathbf{A}, \mathbf{C}=$ Square nonsingular matrix (unnecessarily identical sizes)

$\mathbf{B}, \mathbf{D}=$ Matrix of compatible sizes

There are following sequence of steps of adaptive RLS algorithm: at the receipt of new $\mathbf{u}(k)$ data the signal filtration is performed usage of $\mathbf{w}(k-1)$ filter current coefficients and error size calculation of exemplary signal recreation: $y(k)=\mathbf{u}^{\mathrm{T}}(k) \mathbf{w}(\boldsymbol{k}-\mathbf{1}), \mathbf{e}(k)=d(k)-y(k)$. The vector-column of effective gain is being settled up (it should be noted that in the next formula a common denominator is a scalar, but not matrix):

$$
\mathbf{K}(k)=\frac{\mathbf{P}(k-1) \mathbf{u}(k)}{1+\mathbf{u}^{\mathrm{T}}(k) \mathbf{P}(k-1) \mathbf{u}(k)},
$$

Evaluation upgrade of inverse correlation signal matrix is held:

$$
\mathbf{P}(k)=\boldsymbol{P}(k-1)-\mathbf{K}(k) \mathbf{u}^{\mathrm{T}}(k) \mathbf{P}(k-1),
$$

Filter coefficients upgrading is used:

$$
\mathbf{w}(k)=\mathbf{w}(k-1)+\mathbf{K}(k) \mathrm{e}(k),
$$

Analysis of error signals for the adaptation algorithms is conducted in Figure 6. A result shows that LMS (Least Mean Square) - an algorithm convergences slowly enough and has substantial enough remaining noises. RLS and Kalman algorithms give better indexes and coincide more quickly [12, 20, 25]. 


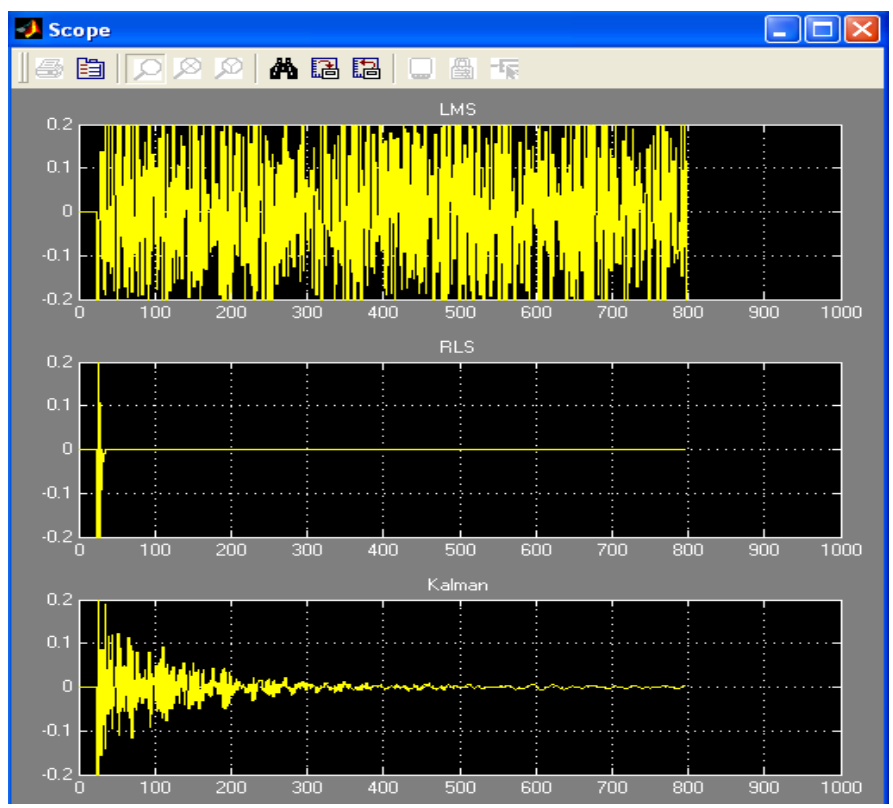

Figure 6. Signals of error for three adaptation algorithms

Thus, the primary purpose of adaptive coding is to provide the necessary data transmission validity at minimum code redundancy. Such coding allows to extend the information transfer rate or decrease the emission frequency band due to adaptation of the used coder to the telecommunication channel state. The idea of adaptation lies in the fact that a receiver estimates SNR in a telecommunication channel and on the basis of this estimation chooses from the certain totality a code which has high code rate and provides the set validity. The process is directly connected with a choice of digital modulation mode [5, 11, 26].

\subsection{Noise immunity study a multi-threshold decoder}

Figure 7 and Figure 8. show the noise immunity curves of multi-threshold decoder under conditions of adaptive decoding algorithm application by a multi-threshold decoder in a telecommunication channel with SCC on the BPSK and QPSK basis for providing of error probability decoding at the 10E-6 level [11]. Results of noise immunity researching of multi-threshold decoder with mentioned codes are shown in Figure 9.

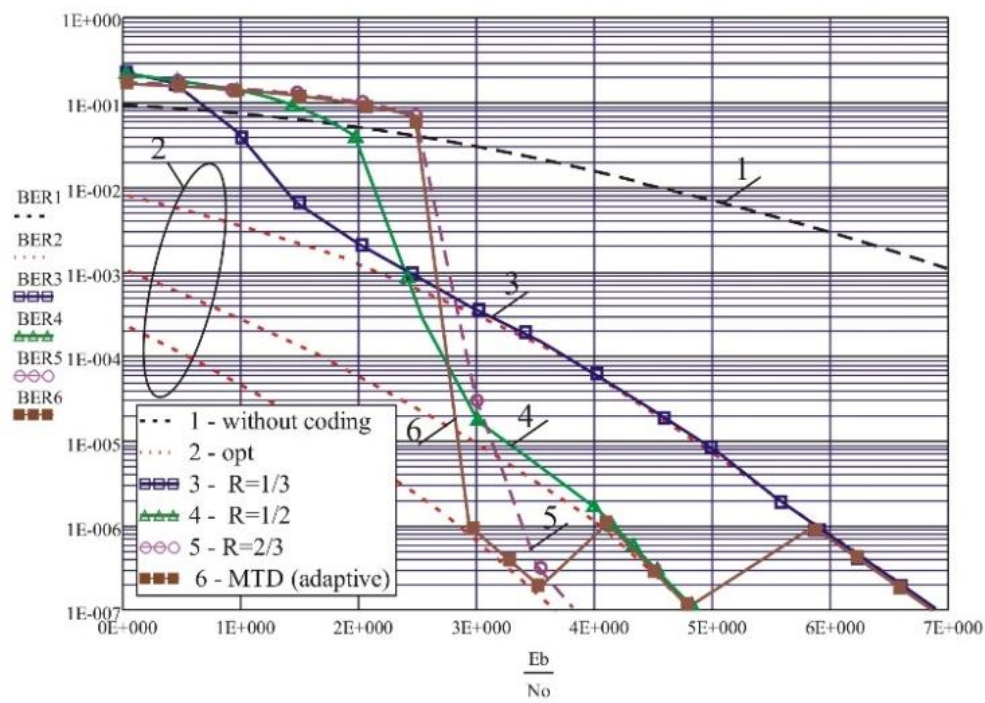

Figure 7. Specifications of noise immunity of adaptive multi-threshold decoder in AGWN- channel for long block codes 
There is satisfactory approaching of noise immunity curve to optimum decoder curve (1-opt). Formed a block self-orthogonal code at $R=4 / 12$ rate and by $d=17$ code distance, length 100000 bits. Formed codes with 4/6, 4/7, 4/8, 4/9, 4/10, 4/11 rate, with 3, 5, 7, 9, 11, 13, 15 code distances. Used the described RLS algorithm [27-29] for realization of the adaptive decoding.

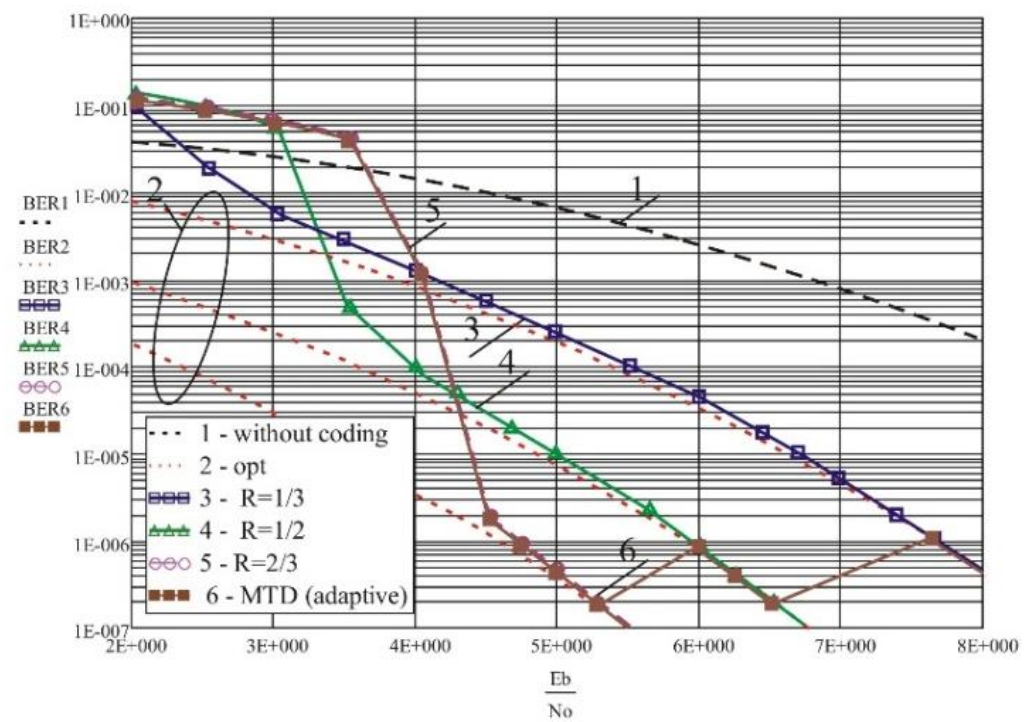

Figure 8. Specifications of noise immunity of adaptive multi-threshold decoder (BPSK) for long convolved codes

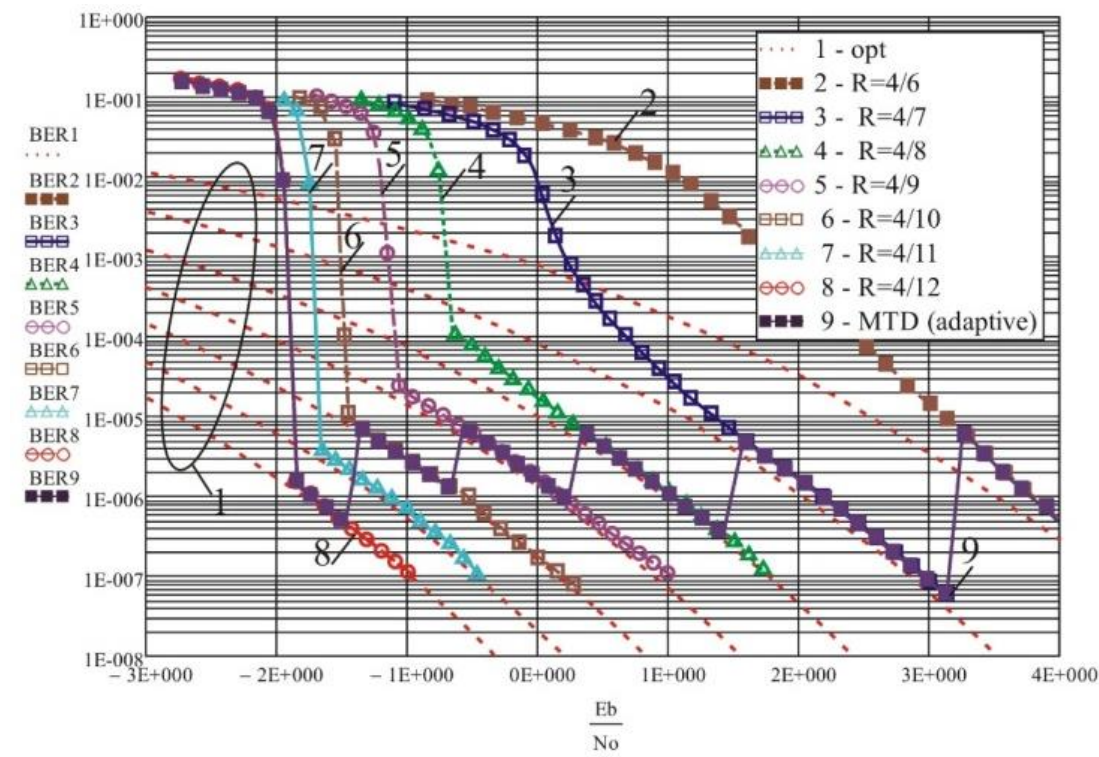

Figure 9. Specifications of noise immunity of adaptive multi-threshold decoder (QPSK) of block self-orthogonal codes

The adaptive coding [30] algorithm foresees the limits selection process of SNR relation and each of investigational codes provides the necessary descriptions and owns with minimum surplus in its limits. The key point of adaptive coding is in the management of multi-threshold decoder parameters adaptively. Such approach allows to save the frequencies band and get rate gain. Powerful noises channel will induce powerful code application with lots of decoding iterations. Opposite, insignificant noises channel modifies a decoder to the state which is determined less of iterations at decoding. 


\section{CONCLUSION}

Solution of the problem received through formation of signal-code construction designs which combined contradictory properties of "dense" multiposition signals (high frequency efficiency) and noise immunity codes (high energy efficiency) in a single design, which provided simultaneous increase in both frequency and energy efficiency the processing of signals in telecommunications facilities. For the task decision of probability diminishing of decoding error for self-orthogonal codes in the band of their nearly optimal decoding scheme modification of multi-threshold decoder is offered by the use of filtration adaptive algorithms.

The adaptive control of work mechanism of multi-threshold decoder efficiency is offered by the redundancy connecting management or checking spurs cutover in a decoder structure. Checking spurs shutting down and connecting can get the set of data transmission validity at minimum code surplus without the coder and decoder changes in case of application of adaptive decoding algorithm by a multi-threshold decoder in a telecommunication channel with SCC on the basis of BPSK and QPSK for providing of error probability decoding at $10 \mathrm{E}-6$ level is shown.

This analysis makes it possible to establish that the multi-threshold decoder application for decoding of short codes practically does not influence on the increase of CG relative to a threshold decoder. Codes usage with the small effect of errors propagation and adaptive decoder provides decoding which tends to be optimum and allows to increase $\mathrm{CG}$ to $2,5 \mathrm{~dB}(\mathrm{BER}=10 \mathrm{E}-6)$. As well, researches have illustrated that multi-threshold decoder at identical efficiency with turbo codes will be about $10 \div 100$ times quicker.

\section{REFERENCES}

[1] Y. Liu, et al., "Multi-Threshold Bit Flipping Algorithm For Decoding Structured LDPC Codes," IEEE Communications Letters, vol. 19, no. 2, pp. 127-130, Feb 2015.

[2] J. Boiko and O. Eromenko, "Signal Processing in Telecommunications with Forward Correction of Errors," Indonesian Journal of Electrical Engineering and Computer Science, vol. 11, no. 3, pp. 868-877, 2018.

[3] R. W. Middlestead, "Forward Error Correction Coding Without Bandwidth Expansion," in Digital Communications with Emphasis on Data Modems: Theory, Analysis, Design, Simulation, Testing, and Applications, Wiley, pp. 339-373, 2017.

[4] M. Y. Kushnir, et al., "Information security of the chaotic communication systems," in 8th CHAOS Conference Proceedings, pp. 441-452, 2015.

[5] R. K. Mohammed and H. A. Abdullah, "Implementation of digital and analog modulation systems using FPGA," Indonesian Journal of Electrical Engineering and Computer Science, vol. 18, no. 1, pp. 485-493, 2019.

[6] J. Boiko, et al., "Productivity of telecommunication systems with modified signal-code constructions," in 20174 th International Scientific-Practical Conference Problems of Infocommunications. Science and Technology, (PIC S\&T), pp. 173-178, 2017.

[7] N. El Maammar, et al., "A comparative simulation study of different decoding schemes in LDPC coded OFDM systems for NB-PLC channel," Indonesian Journal of Electrical Engineering and Computer Science, vol. 15, no. 1, pp. 306-313, Jul 2019.

[8] N. Telagam, et al., "BER analysis of concatenated levels of encoding in GFDM system using labview," Indonesian Journal of Electrical Engineering and Computer Science, vol. 14, no. 1, pp. 77-87, Apr 2019.

[9] I. A. Joundan, et al., "A new efficient way based on special stabilizer multiplier permutations to attack the hardness of the minimum weight search problem for large BCH codes," International Journal of Electrical and Computer Engineering, vol. 9, no. 2, pp. 1232-1239, Apr 2019.

[10] M. Fadhil, et al., "Maximizing signal to leakage ratios in MIMO BCH cooperative beamforming scheme," International Journal of Electrical and Computer Engineering, vol. 9, no. 5, pp. 3701-3713, Oct 2019.

[11] J. Boiko, et al., "Signal processing with frequency and phase shift keying modulation in telecommunications," TELKOMNIKA Telecommunication Computing Electronics and Control, vol. 17, no 4, pp. 2025-2038, Aug 2019.

[12] B. C. Civek, et al., "Big data signal processing using boosted RLS algorithm," in 2016 24th Signal Processing and Communication Application Conference (SIU), pp. 1089-1092, 2016.

[13] H. Mu, et al., "Weighted Symbol Flipping Decoding for Non-binary LDPC Codes Based on Iteration Stopping Criterion," in 2018 37th Chinese Control Conference (CCC), pp. 8447-8452, 2018.

[14] V. Zolotarev, et al., "Effective multithreshold decoding algorithms for wireless communication channels," in 2016 IEEE 10th International Conference on Application of Information and Communication Technologies (AICT), pp. 1-5, 2016.

[15] N. Grinchenko, et al., "Improving performance of multithreshold decoder over binary erasure channel," in $20176 t h$ Mediterranean Conference on Embedded Computing (MECO), pp. 1-4, 2017.

[16] C. Lahsini and A. Bouallegue, "Channel decoding of Reed Solomon block turbo codes with a priori information : Application to image transmission," in 2017 International Conference on Wireless Networks and Mobile Communications (WINCOM), pp. 1-6, 2017.

[17] G. M. Kraidy, "On Progressive Edge-Growth Interleavers for Turbo Codes," IEEE Communications Letters, vol. 20, no. 2, pp. 200-203, Feb 2016. 
[18] Salija P. and Yamuna B., "Implementation of Turbo Code with Early Iteration Termination in GNU Radio," Journal of Telecommunication, Electronic and Computer Engineering, vol. 9, no. 2, pp. 53-59, Jun 2017.

[19] M. Ahmed, et al., "Performance Comparison of Energy Efficiency and Physical Layer Security for Reed Solomon and Bose-Chaudhuri-Hocquenghem Codes in Wireless Sensor Networks," in 2018 7th International Conference on Computer and Communication Engineering (ICCCE), pp. 157-160, 2018.

[20] S. N. A. M. Amin, et al., "Kalman Filter Estimation of Impedance Parameters for Medium Transmission Line," TELKOMNIKA Telecommunication Computing Electronics and Control, vol. 16, no 2, pp. 900-908, Apr 2018.

[21] S. Alkurwy, "A novel approach of multiplier design based on BCD decoder," Indonesian Journal of Electrical Engineering and Computer Science, vol. 14, no. 1, pp. 306-313, Apr 2019.

[22] Y. M. Boiko and R. O. Boryachok, "Improving effectiveness for processing signals in data transmission channels with phase manipulation," in 2013 23rd International Crimean Conference Microwave \& Telecommunication Technology, pp. 262-263, 2013.

[23] M. A. R. Anjum, “A New Approach to Adaptive Signal Processing," Indonesian Journal of Electrical Engineering and Informatics, vol. 3, no. 2, pp. 93-108, Jun 2015.

[24] S. M. Sulong, et al., "Performance Evaluation of Adaptive Algorithm with Linear Equalizer in MIMO OFDMA System," Journal of Telecommunication, Electronic and Computer Engineering, vol. 9, no. 1-3, pp. 129-132, 2018.

[25] A. Azwan, et al., "A multiobjective simulated Kalman filter optimization algorithm," in 2018 IEEE International Conference on Applied System Invention (ICASI), pp. 23-26, 2018.

[26] H. Yoshikawa, "On the bit error probability for constant log-MAP decoding of convolutional codes," in 2016 International Symposium on Information Theory and Its Applications (ISITA), pp. 502-506, 2016.

[27] V. H. Nascimento and Y. V. Zakharov, "RLS Adaptive Filter With Inequality Constraints," in IEEE Signal Processing Letters, vol. 23, no. 5, pp. 752-756, May 2016.

[28] D. Sun, et al., "Recursive regularisation parameter selection for sparse RLS algorithm," in Electronics Letters, vol. 54, no. 5, pp. 286-287, 2018.

[29] S. Dixit and D. Nagaria, "LMS Adaptive Filters for Noise Cancellation: A Review," International Journal of Electrical and Computer Engineering, vol. 7, no. 5, pp. 2520-2529, Oct 2017.

[30] F. Ye, et al., "Optimized Rate-Adaptive Protograph-Based LDPC Codes for Source Coding With Side Information," in IEEE Transactions on Communications, vol. 67, no. 6, pp. 3879-3889, Jun 2019. 